ISSN-PRINT 1794-9831 / E-ISSN 2322-7028

Vol. 18 No 3 / sep - dic, 2021 / Cúcuta, Colombia.

do $\underline{\text { https://doi.org/10.22463/17949831.2912 }}$

Original

\title{
La experiencia de los cuidadores familiares de personas con cáncer. Estudio fenomenológico
}

The experience of family caregivers of people with cancer. Phenomenological study

A experiência dos cuidadores familiares de pessoas com câncer. Estudo fenomenológico

\author{
Yenny Andrea Giraldo-Toro ${ }^{*}$ \\ Camilo Duque-Ortiz ${ }^{2}$
}

\section{Resumen}

Objetivo: Comprender la experiencia vivida de los cuidadores familiares de personas que padecen enfermedades oncológicas. Materiales y métodos: Estudio de enfoque cualitativo, basado en la fenomenología interpretativa. Se desarrolló en Medellín, Colombia, entre julio de 2018 y junio de 2019. La recolección de la información se realizó a través de entrevistas semiestructuradas realizadas a diez cuidadores familiares de personas con enfermedades oncológicas. El total de los participantes estuvo determinado por la saturación teórica de los temas emergentes. Resultados: La experiencia que vive el cuidador familiar de una persona que padece cáncer, se manifiesta a través de tres temas emergentes: enfrentarse a un nuevo camino, en el que hay situaciones derivadas de la enfermedad que imponen unas cargas y demandan un sobre-esfuerzo al cuidador. Organizarse para cuidar, el cual se refiere a la manera como se organizan a través del establecimiento de roles, rutinas y redes de apoyo, para hacer frente al cuidado del ser querido. Transformaciones para la vida, que se refieren a la manera como la vida cambia a partir de la experiencia de cuidado. Conclusiones: La experiencia que viven los cuidadores familiares de personas con cáncer se interpreta como una vida de entrega hacía su pariente enfermo, en la que el nuevo camino al que se enfrentan, la organización de su vida para cuidarlo y las transformaciones que experimentan, giran en torno a la enfermedad y al cuidado que demanda la persona con cáncer.

Palabras clave: Cuidadores; emociones; adaptación psicológica; costo de enfermedad.

\begin{abstract}
Autor de correspondencia*
${ }^{1 *}$ RN. MSc. Enfermera sala de quimioterapia. Hospital General de Medellín. Medellín, Colombia. Correo: jennytoro@gmail.com (1) 0000-0002-0296$\underline{1089}$.

${ }^{2}$ ERN. Esp. MSc. PhD. Docente titular. Facultad de Enfermería. Universidad Pontificia Bolivariana. Medellín, Colombia. Correo: camilo.duque@upb. edu.co (i) $\underline{0000-0003-3106-0471}$
\end{abstract}

Recibido: 15 marzo 2021

Aprobado: 20 agosto 2021

Para citar este artículo

Giraldo-Toro YA, Duque-Ortiz C. La experiencia de los cuidadores familiares de personas con cáncer. Estudio fenomenológico. Rev. cienc. cuidad. 2021; 18(3):43-53. https:// doi.org/10.22463/17949831.2912

\footnotetext{
Abstract

Objective: Understand the experience of family caregivers of people with oncologic diseases. Materials and methods: Study with a qualitative approach, based on the interpretative phenomenology analysis. It was developed in Medellin, Colombia, between July of 2018 and June of 2019. The recollection of information was made through semi-structured interviews on ten family caregivers of people with oncologic diseases. The total of the participants was determined by the theoretical saturation of the emerging subjects. Results: The experience lived by the family caregiver of a person with cancer, is manifested through three emerging subjects: facing a new way, where there are new situations derived from the disease that impose workloads and demand an over exertion of the caregiver. Organizing to care, which refers to the ways they organize themselves through the establishment of roles, routines and support networks, to cope with the care of loved one. Life transformations, which refers to the way that life changes from the experience of care. Conclusions: The experience of family
}

(C) Universidad Francisco de Paula Santander. Este es un artículo bajo la licencia CC-BY-NC-ND 


\section{Scientific Journal of Nursing}

caregivers of people with cancer is interpreted as a life dedicated to their sick relative, where the new path they face, the organization of their life for caring and the transformations experiences, revolve around the disease and the care demanded by a person with cancer.

Keywords: Caregivers; emotions; psychological adaptation; cost of the disease.

\section{Resumo}

Objetivo: compreender a experiência vivida dos cuidadores familiares de pessoas que padecem doenças oncológicas. Materiais e métodos: estudo qualitativo baseado na fenomenologia interpretativa. Desenvolveu-se em Medellín, Colômbia, entre julho de 2018 e junho de 2019. A informação foi coletada através de entrevistas semiestruturadas realizadas a dez cuidadores familiares de pessoas com doenças oncológicas. O total dos participantes esteve determinado pela saturação teórica dos temas emergentes. Resultados: a experiência que vive o cuidador familiar da pessoa com câncer, manifesta-se através de três temas: enfrentar-se ao novo caminho, em que há situações derivadas da doença que impõem cargas e demandam maior esforço do cuidador. Organizar-se para cuidar, refere-se à maneira como organiza os seus papeis, rutinas e redes de apoio, para fazer frente ao cuidado requerido. Transformações para a vida, maneira como a vida muda a partir da experiência do cuidado. Conclusões: a experiência vivida pelos cuidadores familiares de pessoas com câncer interpreta-se como uma vida de doação ao seu parente doente, na que o novo caminho percorrido, a organização da sua vida para cuidar-lhe e as transformações que experimentam ao redor da doença e ao cuidado que demanda a pessoa com câncer.

Palavras-chave: Cuidadores; adaptação psicológica; emoções; efeitos psicossociais da doença

\section{Introducción}

El cáncer es una enfermedad crónica que, según Wang et al. (1) genera deterioro físico y alteración mental, social, económica y espiritual, que puede llevar a la persona que la padece a perder la capacidad de cuidarse por sí misma y a requerir el apoyo de otros individuos, a quienes se les denomina cuidadores.

La persona que padece cáncer puede presentar un deterioro de su estado de salud, tanto por la enfermedad en sí misma como por el tratamiento que conlleva, hasta el punto de impedirle realizar de manera autónoma, actividades básicas de la vida diaria. De ahí que dichas personas generen una dependencia parcial o total de su cuidador, el cual suele ser familiar (2). De esta manera, el cuidador familiar, además de seguir con su propia vida, asume el cuidado de otra persona, incluso hasta el punto de priorizar las necesidades de estos por encima de las propias (3).

Flores et al. (4) consideran que el cuidador es aquella persona que asiste o cuida a otra que presenta dis- capacidad, minusvalía o incapacidad para el desarrollo normal de sus actividades vitales o relaciones sociales. Dentro de los tipos de cuidadores, el cuidador familiar se define como la persona que tiene un vínculo de parentesco o cercanía y asume la responsabilidad del cuidado de un ser querido (5).

El cuidador familiar, también suele convertirse en el apoyo emocional y económico del paciente. En lo emocional, lo acompaña en el proceso de afrontamiento y contribuye a favorecer la adaptación de la persona con cáncer a los cambios derivados del proceso de la enfermedad $(5,6)$. En lo económico, asume los gastos que se generan el tratamiento y cuidado del paciente. Esto último, hace que el cuidador familiar, deba emprender la búsqueda de nuevos ingresos económicos a través de trabajos adicionales o informales (7).

Adashek y Sibbiah (8) plantean que los cuidadores familiares cambian sus dinámicas personales, laborales, familiares y sociales, pues el cuidado de la persona enferma les impone nuevos retos que contrastan y les impide continuar con sus actividades cotidianas. Thomas-Hebdon et al. (2) y Chaparro et al. (9) expo- 
nen que las nuevas dinámicas a las que se enfrentan los cuidadores familiares, si bien, están orientadas a mejorar el bienestar del paciente, suelen producirles alteraciones físicas, mentales y sociales, que pueden llevarlos a una pérdida de rol en su contexto habitual, de las interacciones sociales, dificultades en el trabajo $\mathrm{y}$ una sensación de incomodidad con respecto a la vida. Sarabia et al. (10) refieren que los cuidadores perciben una falta de soporte social y de apoyo de los sistemas de salud. En este sentido, esperan recibir formación y acompañamiento en cuanto al desarrollo de habilidades de cuidado y a la adquisición de conocimientos sobre la enfermedad que padece la persona que cuidan. Así mismo, desean contar con un apoyo emocional que contribuya a afrontar la experiencia de ser cuidador.

Por otra parte, la asociación que se hace del cáncer con la muerte, su aparición abrupta y tempestiva, el alto costo que genera y el impacto emocional que suele producir en el paciente y su familia, pueden llevar a que la experiencia de cuidar a una persona con cáncer tenga una realidad y significados diferentes que cuando se cuidan personas con otras enfermedades crónicas $(2$, $3,6-9,11)$.

Xiaoyum y Fenglan (12) expresan que es importante que se conozcan las experiencias de la vivencia del cuidador en situaciones crónicas de enfermedad, para de acuerdo a ello, tener una base y una aproximación a la realidad que perciben estas personas. De igual forma, Wyatt et al. (13) proponen que se debe obtener una mejor comprensión de la experiencia del cuidador familiar de la persona con cáncer. A partir de lo expuesto, el objetivo del presente estudio es comprender la experiencia vivida por los cuidadores familiares de personas con cáncer.

\section{Materiales y métodos}

El presente estudio se desarrolló bajo el enfoque cualitativo de investigación y a partir del método de la fenomenología interpretativa.

Los criterios de inclusión que orientaron la selección de los participantes fueron los siguientes: personas que, por parentesco o afiliación, fueran reconocidas por los enfermos con cáncer como su familiar y que, a su vez, lo aceptaran como sus cuidadores; que el paciente fuera un adulto mayor de 18 años y que padeciera cáncer, sin importar el tipo y estadio del tratamiento.

La búsqueda de los participantes se efectuó por medio de un muestreo a conveniencia y bola de nieve. La selección se orientó por un teórico y el total de participantes se determinó por la saturación de los temas emergentes.

Además, se contó con la participación de diez cuidadores familiares, los cuales se contactaron de dos formas: a) por medio de colegas, amigos y familiares con quienes se localizaron siete participantes; b) a través de una fundación para la atención de pacientes con cáncer, de donde se identificaron y contactaron a tres cuidadores familiares. Todos los participantes invitados aceptaron colaborar en la investigación.

El estudio se realizó en la ciudad de Medellín, Colombia. El periodo de recolección de la información fue entre julio de 2018 y junio de 2019. Para la técnica de recolección de datos se utilizó la entrevista semiestructurada. Las entrevistas tuvieron una duración aproximada entre 45 a 90 minutos y las hizo la investigadora principal. Igualmente, se solicitó consentimiento informado previo, a los entrevistados. Los lugares de las entrevistas fueron acordados con los participantes, privilegiando el lugar donde se sintieran cómodos. En la mayoría de los casos, se hicieron en el hogar del entrevistado y en otros casos, los participantes lo realizaron en las instituciones de salud donde se encontraban cuidando a su ser querido. En este último caso, la entrevista se efectuó en un espacio diferente a la habitación del paciente con el propósito de conservar la privacidad.

Las entrevistas se estructuraron en torno a una guía de cinco preguntas orientadas a obtener información sobre: cómo era un día cuidando a su familiar, las relaciones con su familia y el paciente, los aprendizajes que se obtienen durante el proceso de cuidado y los cambios experimentados desde que se inició el cuidado del pariente enfermo.

Las entrevistas se grabaron en un dispositivo de audio y se contó con un diario de campo en el cual se registraron actitudes, repuestas emocionales o aspectos que iban surgiendo. Se debe precisar, que los datos anteriores posibilitaron la formulación de nuevas preguntas que permitieron profundizar y ampliar sobre la experiencia vivida por el cuidador. Posteriormente se transcribieron en un procesador de texto y se asignaron pseudónimos a cada participante para garantizar la confidencialidad de la información.

El análisis de los datos se desarrolló mediante el programa Excel y se orientó por medio del Análisis Fenomenológico Interpretativo - (Interpretative Phenomenological Analysis- IPA). El IPA fue descrito por Smith (14) como un proceso que es doblemente hermenéutico o interpretativo, ya que permite a los participantes dar sentido a su mundo y al investigador le facilita su codificación e interpretación. El proceso de análisis se 
desarrolló en tres fases, las cuales se describen en la Tabla 1

Tabla 1. Fases del proceso de análisis.

\begin{tabular}{cl}
\hline Fases & \multicolumn{1}{c}{ Descripción } \\
\hline 1 & $\begin{array}{l}\text { Precodificación de cada entrevista, a partir de una línea- por línea, de las trascripciones. Anotación de } \\
\text { reflexiones o códigos, al margen del texto. }\end{array}$ \\
& Lectura entre dos y tres veces, de cada entrevista. Ajuste de notas y códigos en cada relectura. \\
2 & $\begin{array}{l}\text { Identificación de Unidades de Análisis (fragmentos de entrevistas) y asignación de un código. En algunos } \\
\text { casos, se conservó los códigos del paso anterior y en otros casos se hizo una recodificación. }\end{array}$ \\
$3 \quad$ & $\begin{array}{l}\text { Identificación de semejanzas conceptuales entre los códigos. Agrupación de esto en denominaciones con- } \\
\text { ceptuales más amplias, llamados subtemas. } \\
\text { Los subtemas se agruparon en temas. } \\
\text { La estructuración jerárquica conceptual a través de los temas y subtemas permitió representar las experien- } \\
\text { cias vividas de los cuidadores familiares de personas con cáncer y oriento la escritura y descripción de los } \\
\text { hallazgos que emergieron del análisis. }\end{array}$
\end{tabular}

Fuente: Aplicación estrategia Análisis Interpretativo Fenomenológico- IPA

El análisis de los datos se orientó por muestreo teórico, para esto se programaron periodos de recolección de información seguidos de periodos de análisis. Para apoyar el proceso de análisis se utilizaron diagramas y memos analíticos y teóricos.
Para darle rigor científico al estudio se tuvieron en cuenta los criterios de credibilidad, auditabilidad y transferibilidad, como se describen en la Tabla 2

Tabla 2. Criterios de rigor científico del estudio

\begin{tabular}{cl}
\hline \multicolumn{1}{c}{ Criterio } & \multicolumn{1}{c}{ Descripción } \\
\hline Credibilidad & $\begin{array}{l}\text { Se realizó un proceso de reflexividad constante tanto durante la formulación del proyecto } \\
\text { como el proceso de recolección de información y análisis. } \\
\text { La investigadora principal realizó las entrevistas, transcripción, análisis e interpretación } \\
\text { de los datos. }\end{array}$ \\
& $\begin{array}{l}\text { Se contó con el acompanado de un asesor metodológico quien oriento el proceso de for- } \\
\text { mulación del proyecto, recolección de información, análisis e interpretación. }\end{array}$ \\
& $\begin{array}{l}\text { Las entrevistas fueron grabadas en un dispositivo de audio y transcritas en un procesador } \\
\text { de texto. }\end{array}$ \\
& $\begin{array}{l}\text { Se hizo un proceso de validación de resultados con los participantes } \\
\text { Auditabilidad }\end{array}$ \\
& $\begin{array}{l}\text { Se realizaron talleres de procesos metodológicos con otros investigadores. } \\
\text { Se realizaron presentaciones a comunidades académicas. }\end{array}$ \\
& Se sometió el proyecto a evaluación por Comité de Ética. \\
Transferibilidad & Se hizo un análisis y reflexión sobre la aplicabilidad de los resultados en otros escenarios. \\
\hline
\end{tabular}

Fuente: análisis y desarrollo de los criterios de rigor de la investigación cualitativa.

El estudio se presentó al Comité de Ética de Investigación en Salud de la Universidad Pontificia Bolivariana de la ciudad de Medellín, Colombia, el cual concedió el aval para su ejecución mediante el acta No. 10 del 25 de junio de 2018 .

\section{Resultados}

En la Tabla 3 se describen las características sociodemográficas de los participantes. Como se observa, predominaron las mujeres, con una edad media de 40 años. Según el grado de parentesco, la mayoría de los cuidadores familiares fueron hijos. 
Tabla 3. Características de los participantes

\begin{tabular}{|c|c|c|c|c|c|c|c|}
\hline \multicolumn{4}{|c|}{ Características del cuidador } & \multicolumn{4}{|c|}{ Características de la persona cuidada } \\
\hline $\begin{array}{l}\text { Partici- } \\
\text { pante }\end{array}$ & Genero & Edad & Parentesco & Edad & Tipo de familia & Tipo de cáncer & Tratamiento \\
\hline E01 & Femenino & 31 & Hija & 50 & Nuclear & Cáncer de seno & Cuidado paliativo \\
\hline E02 & Femenino & 30 & Hija & 57 & Monoparenteral & Cáncer de seno & $\begin{array}{l}\text { Quimioterapia am- } \\
\text { bulatoria }\end{array}$ \\
\hline E03 & Masculino & 35 & Hijo & 55 & Monoparenteral & Cáncer de pulmón & $\begin{array}{c}\text { Quimioterapia am- } \\
\text { bulatoria }\end{array}$ \\
\hline E04 & Femenino & 45 & Esposa & 60 & Nuclear & $\begin{array}{l}\text { Leucemia mieloide } \\
\text { crónica }\end{array}$ & $\begin{array}{l}\text { Quimioterapia hos- } \\
\text { pitalaria }\end{array}$ \\
\hline E05 & Femenino & 35 & Hija & 63 & Monoparenteral & Cáncer de páncreas & Cuidado paliativo \\
\hline E06 & Femenino & 45 & Madre & 25 & Monoparenteral & $\begin{array}{l}\text { Leucemia linfo- } \\
\text { blástica aguda }\end{array}$ & $\begin{array}{l}\text { Quimioterapia hos- } \\
\text { pitalaria }\end{array}$ \\
\hline E07 & Femenino & 40 & Madre & 18 & Monoparenteral & $\begin{array}{l}\text { Leucemia linfo- } \\
\text { blástica aguda }\end{array}$ & $\begin{array}{l}\text { Quimioterapia hos- } \\
\text { pitalaria }\end{array}$ \\
\hline E08 & Femenino & 45 & Madre & 18 & Compuesta & $\begin{array}{l}\text { Leucemia linfo- } \\
\text { blástica aguda }\end{array}$ & $\begin{array}{l}\text { Quimioterapia hos- } \\
\text { pitalaria }\end{array}$ \\
\hline E09 & Femenino & 50 & Hermana & 45 & Nuclear & Cáncer de seno & Seguimiento \\
\hline E10 & Masculino & 48 & Hermano & 60 & Extensa & Mieloma múltiple & $\begin{array}{l}\text { Quimioterapia hos- } \\
\text { pitalaria }\end{array}$ \\
\hline
\end{tabular}

Fuente: A partir de los datos sociodemográficos de los participantes

Del análisis de los datos, se identificó que los cuidadores familiares de personas con cáncer se enfrentan a un nuevo camino, se organizan para cuidar a su ser querido y sufren transformaciones para el resto de la vida.

\section{Enfrentarse a un nuevo camino}

Cuando se conoce la noticia de que el ser querido tiene cáncer, se marca un punto de quiebre en la vida del cuidador familiar, pues todo empieza a ser diferente. A partir de este momento se enfrenta a nuevos retos, dinámicas y situaciones derivadas del cuidado, que se perciben como cargas que influyen a nivel físico, mental, emocional y social que, si se prolongan, pueden conducirlo a un estado de agotamiento o síndrome del cuidador. En este sentido, dichas cargas corresponden al costo personal que le impone al cuidador la enfermedad del cáncer que padece el familiar.

Para el cuidador familiar su vida deja de ser la misma, incluso, llega a considerar que ésta deja de ser suya, pues se apaga la luz que alumbra su camino para dar paso a uno nuevo que lo lleva a reconocerse como alguien totalmente diferente, como lo refiere el siguiente cuidador: "A uno se le apaga la vida, ya uno no es lo mismo" (E04). El nuevo camino que toma el cuidador familiar, se caracteriza por una entrega hacía su pariente enfermo, hasta el punto de descuidarse y olvidarse de sí mismo, como lo reconoce el siguiente participante: “(...) estaba pues, en esa parte de descuidán- dome totalmente, pues (...) como de mi" (E02). Tal como lo relata uno de los cuidadores familiares, estos cambios están acompañados de pérdida de concentración, cambios en el estado de ánimo y melancolía: "Obviamente el ánimo, el ánimo cambia, entonces obviamente había, pues (...) hay más llanto, más estados de ánimo bajos, de melancolía" (E02).

Por otra parte, a nivel físico, el cuidador percibe una disminución y desgaste de su estado general de salud asociado a cambios como insomnio, fatiga, molestias musculo-esqueléticas e inapetencia, pues el cuidado de la persona con cáncer demanda esfuerzo físico y de tiempo, como lo describe el siguiente participante: "Es bastante desgastante porque finalmente (...) digamos (...) un enfermo pues demanda esfuerzos, demanda tiempo" (E03). Dicho desgaste puede expresarse en una pérdida significativa y abrupta de peso, como lo relata el siguiente cuidador: "Yo me siento en la ropa (...) me queda grande. De pronto, de pronto, perdi por ahí unos seis kilos" (E10).

La imagen del cáncer como signo de muerte contribuye a que el cuidador familiar perciba, de manera simultánea, múltiples emociones, pensamientos y expectativas, los cuales suelen ser negativos y catastróficos sobre el futuro que le deparará a su pariente enfermo, como lo reconoce el siguiente cuidador: “(...) uno decía... ;cáncer!... y era ya la muerte (...)" (E08). En este sentido, la sola palabra "cáncer" genera miedo e incertidumbre, como se observa en el 
CienciäoChidado

Scientific Journal of Nursing

siguiente relato: “(...) el hecho de que digan cáncer eso es (...) la palabra cáncer asusta” (E09).

Dentro de las emociones que percibe el cuidador familiar se encuentran: la tristeza, el miedo, el llanto, la rabia, la derrota, la incertidumbre y la frustración. Dichas emociones, intensas y desgastantes, se asocian con la incongruencia entre la actitud compasiva del cuidador familiar hacía la persona enferma y la incapacidad de darle confort y solucionarle todo lo que le aqueja, como lo describe el siguiente cuidador familiar: "Las estadías dificiles para mi (...) es verlo en las condiciones en que él se manifiesta, por ejemplo, dolores agresivos, lo hacen llorar, lo hacen poner totalmente incómodo y él me pide que le quite ese dolor, que se lo ayude a quitar (...) ¿cómo hago? (...) no puedo, no hay cómo, no, eso tendría uno que ser mago. Para mí, yo resistir eso (...) imagínese eso (...) un arma traspasándome a mi” (E10).

Por tanto, el cuidado de la persona con cáncer se constituye en un reto, pues el cuidador familiar ejerce su rol en medio de una situación que se enmarca en la desilusión, el deterioro y la progresión de su pariente enfermo hacía la muerte, como lo reconoce el siguiente cuidador familiar: “(...) se les va acabando la vida, como sufren con el dolor, como se van acabando las ganas de vivir, como se deteriora su cuerpo (llanto y silencio), como quieren dejar de vivir" (E01).

En el ámbito social, el cuidador familiar suele cambiar actividades de ocio y recreo para dedicarse a cuidar al ser querido enfermo, como se observa en el siguiente testimonio: "Pero yo qué salir con los amigos o qué ha comer no, yo prefería estar en mi casa" (E01). Estas actividades se disminuyen porque el cuidador tiene poco tiempo para realizarlas, como lo relata el siguiente participante: “(...) me he sentido un poco más limitado porque obviamente (...) digamos, se restringe uno (...) digamos de llegar más tarde, digamos a la $\operatorname{casa}(\ldots)$ (..) (E03).

De igual forma, el alejarse paulatinamente de la vida social puede llevar al cuidador a aislarse socialmente, como lo menciona el siguiente cuidador: "(...) inicialmente yo pues me aislé mucho, aún, aún” (E02). Al principio se alejan de sus propias familias, después de sus amistades negándose a relacionarse con otras personas, como lo describe el siguiente participante: "Me he alejado mucho de la familia" (E04,); “(...) igual yo trabajaba, entonces yo tenía un grupo de amigas allá pues y ya no es lo mismo" (E08).

\section{Organizarse para cuidar}

El rol de cuidador, suele ser nuevo y desconocido, lo que demanda dedicación y esfuerzo para aprenderlo, lo cual debe hacerse en poco tiempo y con recursos propios. Por las circunstancias en las que se presenta, se debe compaginar simultáneamente con los roles que tenía previamente, como se observa en los siguientes relatos: "Mientras también organizaba la casa, estaban ya mis dos sobrinas acá, entonces, yo las cuidaba y bueno (...) todo lo de la casa"(E01); "(...) siento que ahora mi prioridad es la universidad y mi mamá” (E02).
Es por eso, que para lograr una organización de sus actividades, los cuidadores familiares, crean nuevas rutinas y establecen redes de apoyo que les posibiliten atender a su pariente enfermo y continuar con su propia vida.

En relación con las rutinas, se las debe entender como actividades que tienen un desarrollo similar y se hacen de manera repetitiva, lo cual permite acomodarse y disminuir la complejidad que impone el día a día del cuidado del ser querido enfermo. Hay unas rutinas de preparación, las cuales se inician desde temprano, antes de reunirse con el paciente y tienen como propósito organizar el día, beneficiar a la persona enferma y demás integrantes de la familia. Estas rutinas permiten adelantar las actividades del hogar para luego estar a cargo del paciente el resto del tiempo, como lo describe el siguiente participante: “(...) yo siempre me levanto como a las cuatro de la mañana cuando no amanezco acá. Tengo que despacharles, pues, el almuerzo a ellos y ya me preparo para venirme todo el día para acá” (E08).

Otro rasgo a tener en cuenta es que las rutinas que establece el cuidador para el resto del día, dependen del lugar donde se encuentre la persona con cáncer, sea el hospital o el hogar. Conviene subrayar que en el hogar, el cuidador familiar dedica mayor tiempo a labores domésticas como: organizar la casa, preparar los alimentos, higienizar la ropa y brindar confort, como se observa en el siguiente testimonio: "¿Una rutina de todos los días? (...) mijita (...) hacer, asear la casa (...) barrer (...)" (E07). Dichas labores cumplen el objetivo de hacer de la casa un ambiente adecuado para el paciente, que sea cómodo, agradable y, principalmente, limpio, como lo relata el siguiente cuidador familiar: "Las comidas también yo trato de hacerlas con agua hervida o agua (...) o sea, mucho cuidado. Ahi mismo que esté, pues ahi mismo dársela, no dejarla que pase mucho tiempo y no comérsela, todo eso" (E08); "Esté pendiente de su ropa, la ropa se la lavo a mano, no la lavo con la de otros, ni con la de nosotros tampoco, siempre aparte" (E07).

Se debe agregar, que las rutinas en el hospital giran en torno a lo que requiera el pariente enfermo, así como en lo que se pueda apoyar y aportar. En algunos casos, asume la función de informar a la enfermera las novedades relacionadas con el tratamiento del paciente, como se observa en el siguiente relato: "Estaba mirando los medicamentos que la enfermera me dijo (...) cuando estuviera en tal numerito (...) para que le avisara" (E06). Además, apoya en el baño, movilización y acicalamiento del pariente enfermo así como de proteger $\mathrm{y}$ verificar el adecuado funcionamiento de los dispositivos que tiene conectados para el tratamiento, como lo indica el siguiente participante: "Sí, me toca taparla, me toca bañarla pues con mucho cuidado (...) que no se vaya a mojar el catéter" (E08).

De manera análoga, es muy importante el establecimiento de redes de apoyo, puesto que facilitan la organización de los cuidadores familiares. Las redes de apoyo están constituidas por parientes, allegados, instituciones y algunos profesionales de la salud. Estas redes de apoyo ayudan a distribuir las cargas que se asocian al cuidado, como se observa en 
el siguiente relato: "Ahi tenía un amigo muy formal de la iglesia de él, que estudia en la universidad y entonces, él (...) pongamos los jueves o los martes, venía y se quedaba con él" (E06). De igual forma, sirven como soporte para minimizar el impacto emocional que causa la experiencia de ser cuidador, ya que la red de apoyo es útil para que el cuidador familiar se exprese, comparta sus angustias, se distraiga y se sienta motivado para continuar con su rol, como lo relata el siguiente participante: "Gracias a Dios lo tenía a él como mi apoyo, fue un apoyo mucho, muy grande en ese momento, porque si yo no lo hubiera tenido a él, yo ahi sí me hubiera (...) jenloquecido!" (E05). En este sentido, la red de apoyo ayuda al cuidador a tener fuerzas en tiempos difíciles y recibir ánimos para seguir adelante.

\section{Transformaciones para la vida}

La vida del cuidador familiar sufre unos cambios que transforman la manera de pensar, de actuar y de hacer frente a las circunstancias de la vida.

Estas transformaciones para la vida se relacionan con los aprendizajes que se obtienen al cuidar a su ser querido enfermo, como lo describe el siguiente cuidador familiar: "(...) lo veo más es como un aprendizaje, pues son aprendizajes diarios, hay días duros, otros no tanto, pero es más como un aprendizaje" (E02). Estos aprendizajes motivan al cuidador a cambiar la perspectiva de ver la vida y la manera de actuar en esta, como se observa en el siguiente testimonio: "Es una experiencia que te (...) bueno, me tocó con la hija, pues eso no, no lo quiere uno con el hijo, pues yo creo una experiencia que también uno aprende de la vida, aprende a (...) querer uno como que (...) a tenerle más aprecio a la vida" (E07).

Del mismo modo los cuidadores familiares reconocen la experiencia de cuidar a una persona con cáncer como algo difícil que lleva a reflexionar sobre su propia vida, su posición en el mundo y su forma de enfrentarse a las adversidades. De esta manera, la ven como un momento de verdad que los define y determina quiénes son las personas que están a su alrededor, como lo menciona el siguiente cuidador familiar: "(...) uno aprende a vivir, a manejarse y a reconocer quién es quién. Sí, porque vuelve y juega, pues en lo, en lo bueno todo el mundo está, en lo duro es donde uno sabe quién es quién" (E10).

Como se ha dicho las relaciones con el ser querido enfermo también sufren transformaciones, ya que es una experiencia que viven juntos en todo momento, lo cual contribuye a que el vínculo sea más estrecho y cercano, incluso, cuando las relaciones han sido difíciles en el pasado, como se observa en el siguiente relato: “(...) fue una experiencia muy linda porque me acerqué mucho más a ella, me acerqué mucho, mucho más a ella y ella a mí y se apegó de mí (...)” (E05).

Hay que mencionar, además, que surge una relación entre el pariente y el enfermo donde hay una gran demostración de afecto, gratitud, empatía y unidad que surgen y se fortalecen día a día, como lo relata el siguiente participante: “(...) yo me he sentido orgulloso pues de estar con ella, de poderla servir, de poder andar con ella pa'arriba y pa'abajo, me he sentido alegre, contento" (E03). A través de la interacción constante se logra cultivar la relación entre ambos en una conexión que se fortalece cada vez más con la experiencia de cuidar, como lo describen los siguientes cuidadores familiares: "Le conseguimos una faja térmica pero no, no le servía porque ella decía que lo que quería sentir, era mis manos" (E01); "Nos hacemos pues como digamos cariños, pues (...) nos abrazamos, yo a veces la cojo y le doy besos pues en la cabeza o, en fin" (E03).

Es decir, que la fuerza que adquiere esta relación deja gran satisfacción al cuidador familiar, pues considera que el tiempo que invierte cuidando y apoyando a su pariente enfermo, es un tiempo valioso que recordará para siempre con la conciencia tranquila, como lo relata el siguiente participante:

"Yo compartí mucho tiempo con ella y es tiempo que no va a volver, es tiempo que no va a volver y es un tiempo que yo me gané. De conocerla, de ver su dolor, eso ya nadie, esos recuerdos ya nadie me los va a quitar y sí obviamente me queda la conciencia de que yo estuve todo el tiempo con ella y eso sí, pues no me calma el dolor, pero si me da mucha tranquilidad" (E01)

Por tanto, el cuidador familiar adquiere una serie de actitudes positivas que le permiten ver desde otra perspectiva la situación que enfrenta. Esto lo logra con coraje para continuar la vida y seguir adelante, como se observa en el siguiente testimonio: “(...) muy positivos (...) no, hágale que vamos a salir adelante pues con esto $y$, en fin (...) digamos que en épocas sí, en épocas sídonde he tenido pues que coger coraje, cierto, y hacerle pues pa'delante como se dice" (E03).

Esta manera positiva de enfrentar la enfermedad del pariente enfermo, genera esfuerzo para transformar las emociones poco favorables y minimizarlas para el bien del enfermo $\mathrm{y}$ del cuidador, como lo describe el siguiente participante: “(...) no podemos llorar delante de ella, tenemos que ser fuertes, demostrarle que ella va a ser capaz, de que la vida continúa, mire tantas personas que salen adelante, siguen su vida, siguen siendo funcionales" (E05).

Finalmente, el cuidador familiar otorga un sentido diferente a su vida, pues el hecho de vivir esta experiencia, hace que frente a situaciones similares sus pensamientos y acciones se encaminen a servir y ayudar al otro, como lo describe el siguiente cuidador familiar: “(...) ahora las cosas son diferentes (...) porque es que una cosa es esto cuando tienes a un hijo por ahi por las circunstancias mías, como (...) yo ya dentro a una clínica y yo veo a todo mundo y a mí, yo pienso en ir a ayudar. $O$ si paso por la calle y veo a alguien tirado (...) a mi me dan ganas de, como que de (...) hacer algo por esas personas, eso siento mucha compasión” (E07). Adquirir conciencia de la experiencia que vive el ser querido enfermo hace al cuidador familiar más sensible frente al dolor del otro y lo lleva a comprender mejor a quien sufre, como se observa en el siguiente testimonio: “(...) todos nos 


\section{CienciagoCuidado}

Scientific Journal of Nursing

necesitamos y que todos a la final somos débiles. Yo soy de pronto poderoso porque estoy aliviado y (...) puedo hacer de pronto un favor a una persona que lo está necesitando y yo no lo (...) a ver (...) de pronto mi salud no me deja mirar eso, pero ahora que estoy acá sí hay que mirar mucho eso" (E10).

\section{Discusión}

Mesa et al. (15) y Espinoza et al. (16) consideran que las cargas que recaen sobre un cuidador son consecuencias negativas que se derivan del cuidado. Al respecto, describen que se pueden presentar dos tipos de cargas, la subjetiva, que corresponde a las actitudes y reacciones emocionales ante la experiencia de cuidar; y la objetiva, que hace referencia al grado de cambios en distintos aspectos del ámbito doméstico y de la vida de los cuidadores.

Hay que mencionar que para los cuidadores familiares, del presente estudio, las cargas se imponen desde el momento en que se conoce la noticia de que su pariente tiene cáncer. Esta afirmación coincide con los hallazgos de Hashemi et al. (3) quienes encontraron que los cuidadores al conocer el diagnóstico de cáncer de su pariente, lo asocian con una enfermedad catastrófica y la inminencia de muerte, lo cual contribuye a que el proceso de la enfermedad sea doloroso y difícil de llevar, especialmente porque desconocen lo que significa cuidar a una persona con cáncer y el nuevo rol que les corresponde asumir de manera súbita.

Rezende et al. (17) plantean que las cargas del cuidador pueden llegar a sobrepasar la capacidad física y mental de estos, convirtiendo la labor de cuidar en un evento estresante crónico que desgasta al cuidador hasta el punto de desarrollar el síndrome del cuidador. Dicho síndrome afecta y repercute en todas las esferas de la persona y se caracteriza porque el cuidador puede presentar pérdidas de control personal, alteraciones en la salud física y emocional y repercusiones negativas en aspectos sociales y económicos (18). Lo anterior puede limitar la capacidad para cambiar comportamientos necesarios para desarrollar el rol de cuidador (19). Por otro lado, Mesa et al. (15) encontraron que los cambios más significativos a nivel físico son los problemas relacionados con la fatiga, los cambios en el dormir, el dolor o los cambios en el apetito. Lo cual va de acuerdo con los resultados de este estudio, pues los cuidadores familiares reconocen tener un deterioro en su estado de salud en general, reflejado en insomnio, fatiga, molestias musculo- esqueléticas e inapetencia.

En adición, la carga mental a la que se exponen los cuidadores familiares de personas con cáncer, se relaciona con la percepción de sobrecarga emocional, estrés, miedo, agotamiento, ansiedad y menor satisfacción con la vida (18, 20). En este sentido, Han et al. (21) descubrieron que los cuidadores familiares pueden sentir culpa porque consideran que contribuyeron a que el cáncer apareciera y se preguntan sobre que hubiesen podido hacer para mejorar las cosas. Al respecto, Shannon (22) identificó que la sensación de ser incapaces para atender las necesidades del pariente enfermo de cáncer generó en el cuidador una carga emocional a la hora de atenderlo. Con respecto a lo planteado por este autor, los cuidadores familiares del presente estudio tuvieron dificultades en la concentración y experimentaron cambios frecuentes en el estado de ánimo, llegando a sufrir depresión. En este sentido, Rodríguez et al. (23) reconocieron que el 19\% de las personas enfermas se oponen, incluso con agresividad a sus cuidadores. Dicha agresividad aumenta significativamente la sobrecarga del cuidador.

Mesa et al. (15) determinaron que el cuidador familiar se puede aislar socialmente, lo que favorece la sensación de soledad y abandono, al perder la capacidad de relacionarse, conversar y dividir su sufrimiento (24). Al respecto, Otis y Juarez (25) exponen que, a pesar de contar con el apoyo familiar, los cuidadores familiares pueden continuar reportando sentimientos de mayor aislamiento, lo que puede contribuir a la presencia de la angustia e impactar en su calidad vida.

Hay que precisar que el ser humano, para enfrentar los estímulos del medio en el que se desenvuelve, organiza sus respuestas de acuerdo a sus creencias, opiniones, estereotipos, actitudes y representaciones, lo que genera una actitud que inconscientemente la persona va asumiendo y adquiriendo para hacer frente a los estímulos del ambiente (26). En el caso concreto, de los cuidadores familiares el organizarse es una forma de hacer frente a la experiencia de cuidar a su ser querido enfermo y esto se inicia con el aprendizaje y conocimiento de las tareas y funciones propias de este rol familiar.

Desde otro punto de vista, Hashemi et al. (3) descubrieron que el rol del cuidador familiar de la persona con cáncer, suele asumirse de manera súbita. Así, para los cuidadores familiares es como entrar en el mundo de lo desconocido. En concordancia con los resultados de este estudio, se encontró que los cuidadores familiares adquieren éste rol sin querer, sin preparación, conocimiento o familiaridad con las tareas que deberán realizar. En este sentido, Hashemi et al. (3) exponen que cuidar de un paciente con cáncer se asocia con tareas inesperadas y difíciles que el cuidador debe reconocer y adaptarse. De ahí, que el cuidador familiar deba organizarse para enfrentar las situaciones que se empiezan a presentar y que alteran su cotidianidad.

Al respecto, Fernandes y Angelo (27) precisaron que el proporcionar atención y apoyo específico a la persona dependiente, lo cual es característico del cuidador, corresponde a un nuevo rol que deben aprender y desarrollar. Es por eso, que los autores exponen que el nuevo rol de cuidador, les genera la necesidad de manejar simultáneamente múltiples tareas que les origina peso y conflicto a los que deben hacer frente. Por otra parte, el establecimiento de rutinas por parte del cuidador, surgen como una forma de adaptarse a la multiplicidad de tareas que se derivan del rol de cuidador, lo cual según Morale et al. (28) contribuyen a que, conforme pasa el tiempo, se tome control de la situación. Así mismo, plantean que las rutinas favorecen el desarrollo de una idea más 


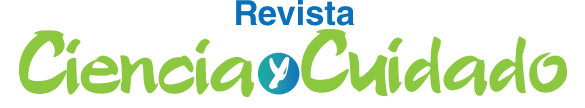

Scientific Journal of Nursing

precisa de las situaciones a las que debe dar la cara, pues el cuidador logra disponer de mayor información, recursos externos y apoyo que se traducen en herramientas para enfrentarse el cuidado de su pariente enfermo.

Precisamente la red de apoyo del cuidado familiar favorece la organización del cuidador en la medida que le permite apoyarse y ser relevado en la ejecución de sus tareas. Para Han et al. (21) la familia se constituye en la principal red de apoyo de los cuidadores familiares, quienes asumen funciones complementarias dependiendo de las situaciones y el contexto en el que se encuentre la persona enferma. Al respecto, Montford et al. (29) precisaron que, en diversas situaciones, los amigos pueden convertirse en una red de apoyo importante para el cuidador familiar, pues estos llegan a ser considerados como parte de la familia. Lo planteado anteriormente, coincide con los resultados del presente estudio, en el que, los cuidadores familiares reconocen que contar con una red de apoyo ayuda a disminuir las cargas del cuidado.

Otro factor a tener muy en cuenta es que las transformaciones para la vida se relacionan con los cambios que experimenta el cuidador familiar, derivados del proceso de aprendizaje de cuidar a la persona enferma con cáncer, el esfuerzo por superar las situaciones adversas y adaptarse a los cambios que trae consigo el proceso de cuidar. Esto contribuye a crear una nueva perspectiva de ver la vida (19).

Del mismo modo la combinación de experiencias de riesgos graves o complejos, como cuidar a una persona con cáncer, y un resultado psicológico relativamente positivo a pesar de esas experiencias, contribuye a desarrollar y fortalecer la capacidad para resistir y recuperarse de desafíos significativos que amenazan la estabilidad y viabilidad de la persona (30). Es por eso., que los cuidadores familiares viven un proceso de acomodación que trasforma sus vidas, ya que, a pesar de enfrentarse ante emociones negativas y cambios radicales, se mantienen en pie para superar las dificultades que se les presentan $(28,31)$.

Por su parte, Martínez y De la Cuesta (32) precisaron que los cuidadores familiares refieren que después de cuidar a un pariente enfermo con cáncer, sus vidas ya no son como antes, ya que a lo largo del tiempo que llevan cuidando, ha ido disminuyendo parte de lo que hacían y tenían, su capacidad y fortaleza están mermadas. Además, sus problemas de salud y la progresiva enfermedad del familiar que cuidan requieren de ellos más esfuerzo y dedicación; por tanto los conduce a pérdidas relacionadas con sus capacidades, su libertad personal y su vida social (16). Sin embargo, el esfuerzo por adaptarse a las dinámicas y situaciones que impone el cuidado y la interacción continua entre el cuidador familiar y el cuidado de su pariente enfermo, contribuyen a sobreponerse con éxito de la difícil experiencia que han vivido $(28,31)$.

En definitiva, las trasformaciones en la vida que realiza el cuidador familiar se reflejan en una vida de entrega a su ser querido enfermo, pues las pérdidas y renuncias que efectúan a lo largo del tiempo que llevan cuidando, cambian una parte significativa de sus vidas, deseos, propósitos y aspiraciones que tenían para sí mismos (32). Esta vida de entrega permite, al cuidador, el fortalecimiento de la relación con su ser querido, el cual coincide con los resultados de Mosher et al. (33) en donde se determina que los cuidadores como sus parientes reportan un cambio positivo común en sus relaciones convirtiéndose en una mayor apreciación de la vida; pues cuidar a una persona con cáncer conduce a una mayor conciencia de la brevedad de la vida..

\section{Conclusiones}

- La experiencia que viven los cuidadores familiares de personas con cáncer se figura como una vida de entrega hacía su pariente enfermo, en la que el nuevo camino al que se enfrentan, la organización de su vida para cuidar y las transformaciones que experimentan se dan en torno a la enfermedad y al cuidado que demanda la persona con cáncer. Este hecho hace que el cuidador familiar renuncie a su propia vida para vivirla conforme a las necesidades y expectativas de su familiar enfermo.

- Los hallazgos obtenidos en el presente estudio aportan insumos que se deben tener muy en cuenta en el desarrollo de políticas públicas en salud relacionadas con la atención y cuidado de personas que padecen enfermedades crónicas como el cáncer; igualmente, hay que incluir a los cuidadores familiares, sin olvidar las experiencias que viven, las cuales, favorecen la creación de una diada entre el cuidador y su pariente para afrontar la enfermedad. De manera idéntica, el cuidador familiar puede constituirse en un apoyo al sistema de salud para el cuidado de la persona enferma con cáncer en el ámbito extrahospitalario y domiciliario.

- Por consiguiente, es pertinente realizar estudios que profundicen en las necesidades más significativas para los cuidadores familiares, pues podría ser un punto de partida para generar mayor conciencia en el cuidado integral del paciente con enfermedad oncológica y su familia. Es por eso, que al incluir los cuidadores familiares en el cuidado del paciente ayuda a disminuir la incertidumbre generada por la enfermedad y también permite a generar estrategias de promoción de la salud que estimulen al cuidador para que también se cuide a sí mismo.

\section{Conflicto de Interees}

Los autores declaran no tener ningún conflicto de intereses. 


\section{Referencias Bibliográficas}

1. Wang L, Shi JF, Zhu J, Huang HY, Bai YN, Liu GX, et al. Health-related quality of life and utility scores of patients with breast neoplasms in China: A multicenter cross-sectional survey. Breast. 2018;39:53-62. https://doi.org/10.1016/j. breast.2018.03.004.

2. Thomas-Hebdon MC, Coombs LA, Reed P, Crane TE, Badger TA. Self-Efficacy in Caregivers of Adults Diagnosed with Cancer: An Integrative Review. Eur J Oncol Nurs. 2021. https://doi.org/10.1016/j.scitotenv.2019.135907

3. Hashemi M, Irajpour A, Taleghani F. Caregivers needing care: the unmet needs of the family caregivers of end-oflife cancer patients. Support Care Cancer. 2018;26(3):759-66.

4. Flores E, Rivas E, Seguel F. Nivel de sobrecarga en el desempeño del rol del cuidador familiar de adulto mayor con dependecia severa. Cienc y Enfermería. 2012;18(1):29-41.

5. Unsar S, Erol O, Ozdemir O. Caregiving burden, depression, and anxiety in family caregivers of patients with cancer. Eur J Oncol Nurs. 2021;50:1-8. https://doi.org/10.1016/j.ejon.2020.101882

6. O'Connor M, Moriarty H, Schneider A, Dowdell EB, Bowles KH. Patients' and caregivers' perspectives in determining discharge readiness from home health. Geriatr Nurs (Minneap). 2021;42(1):151-8. https://doi.org/10.1016/j.gerinurse.2020.12.012

7. Stolz-Baskett P, Taylor C, Glaus A, Ream E. Supporting older adults with chemotherapy treatment: A mixed methods exploration of cancer caregivers' experiences and outcomes. Eur J Oncol Nurs. 2021;50:1-8. https://doi.org/10.1016/j. ejon.2020.101877

8. Adashek JJ, Subbiah IM. Caring for the caregiver: A systematic review characterising the experience of caregivers of older adults with advanced cancers. ESMO Open. 2020;5(5):1-7. https://doi.org/10.1136/esmoopen-2020-000862

9. Chaparro L, Sánchez B, Carrillo GM. Encuesta de caracterización del cuidado de la diada cuidador familiar- persona con enfermedad crónica. Cienc y Cuid. 2014;11(2):31-45.

10. Sarabia-Cobo C, Taltavull-Aparicio JM, Miguélez-Chamorro A, Fernández-Rodríguez A, Ortego-Mate C, Fernández-Peña R. Experiences of caregiving and quality of healthcare among caregivers of patients with complex chronic processes: A qualitative study. Appl Nurs Res. 2020;56:1-7. https://doi.org/10.1016/j.apnr.2020.151344

11. Palacios-Espinosa X, González MI. Representaciones sociales del cáncer y de la quimioterapia en pacientes oncológicos. Diversitas. 2015;10(2):207.

12. Xiaoyun C, Fenglan L. The relationships among insecure attachment, social support and psychological experiences in family caregivers of cancer inpatients. Eur J Oncol Nurs. 2020;44:1-6. https://doi.org/10.1016/j.ejon.2019.101691

13. Wyatt GK, Lehto RH, Sender J. The prevalence and types of complementary and integrative health therapies used by caregivers of patients with cancer. Semin Oncol Nurs. 2019;35(4):342-7. https://doi.org/10.1016/j.soncn.2019.06.005

14. Pietkiewicz I, Smith J. A Practical Guide to Using Interpretative Phenomenological Analysis in Qualitative Research Psychology. Psychol J. 2014;20(1):7-14.

15. Mesa-Gresa P, Ramos-Campos M, Redolat R. Cuidado de pacientes oncológicos: Una revisión sobre el impacto de la situación de estrés crónico y su relación con la personalidad del cuidador y otras variables moduladoras. Psicooncologia. 2017;14(1):93-106.

16. Espinoza-Salgado FS, Reyes-Lagunes I, Velasco-Rojano E, Bravo-González MC, Ramírez Ramírez A. Escala de estrategias de enfrentamiento para cuidadores primarios informales de pacientes con cáncer. Psicooncologia. 2017;14(1):12136.

17. Rezende G, Gomes CA, Rugno FC, Eva G, Lima NKC, De Carlo MMRP. Burden on family caregivers of the elderly in oncologic palliative care. Eur Geriatr Med. 2017;8(4):337-41. http://dx.doi.org/10.1016/j.eurger.2017.06.001

18. Valbuena Castiblanco C, Chaparro Díaz L. Carga del cuidado en la díada en situaciones de cáncer. Psicooncología. 2018;15(2):361-72.

19. McDonnell KK, Owens OL, Hilfinger Messias DAK, Heiney SP, Friedman DB, Campbell C, et al. Health behavior changes in African American family members facing lung cancer: Tensions and compromises. Eur J Oncol Nurs. 2019;38:57-64.

20. Fernández-Sánchez B, Jorge-Marin V, Sánchez-Vázquez CM, Bejar-Alonso E. Atención psicológica para pacientes con cáncer y sus familiares: ¿Qué nos encontramos en la práctica clínica? Psicooncologia. 2016;13(2-3):191-204. 


\section{Scientific Journal of Nursing}

21. Han JH, Han SH, Lee MS, Kwon HJ, Choe K. Primary caregivers' support for female family members with breast or gynecologic cancer. Cancer Nurs. 2016;39(3):49-55.

22. Shannon CS. 'I Was Trapped at Home': Men's Experiences with Leisure While Giving Care to Partners During a Breast Cancer Experience. Leis Sci. 2015;37(2):125-41.

23. Rodríguez-González AM, Rodríguez-Míguez E, Duarte-Pérez A, Díaz-Sanisidro E, Barbosa-Álvarez Á, Clavería A. Estudio observacional transversal de la sobrecarga en cuidadores informales y los determinantes relacionados con la atención a las personas dependientes. Aten Primaria. 2017;49(3):156-65. http://dx.doi.org/10.1016/j.aprim.2016.05.006

24. Sánchez LM, Mabel Carrillo G. Competencia para el cuidado en el hogar diada persona con cáncer en quimioterapia-cuidador familiar. Psicooncologia. 2017;14(1):137-48.

25. Otis-Green S, Juarez G. Enhancing the Social Well-Being of Family Caregivers. Semin Oncol Nurs. 2012;28(4):246-

55.

26. Schulman-Green D, Feder S. Integrating family caregivers into palliative oncology care using the Self- and Family Management Approach. Semin Oncol Nurs. 2018;34(3):252-63.

27. Fernandes CS, Angelo M. Family caregivers: What do they need? An integrative review. Rev da Esc Enferm. 2016;50(4):672-8.

28. Moreale R, Campanella F, Marin F, Skrap M, Palese A. Emotional concerns and coping strategies in Low Grade Glioma patients and reliability of their caregivers in reporting these concerns: Findings from a cross-sectional study. Eur J Oncol Nurs. 2017;30:113-9. https://doi.org/10.1016/j.ejon.2017.08.010

29. Montford KS, Duggleby W, Cumming C, Thomas R, Nekolaichuk C, Ghosh S, et al. "All I can do is help": Transition experiences of male spouse caregivers of women with breast cancer. Can Oncol Nurs J. 2016;26(4):312-7.

30. Hornor G. Resilience. J Pediatr Heal Care. 2017;31(3):384-90.

31. Fernández-Hawrylak M, Hernández-Maillo L, Martínez-García I. Comunicación intra-familiar y cáncer de mama. Psicooncología. 2018;15(1):103-18.

32. Martínez Marcos M, De La Cuesta Benjumea C. La experiencia del cuidado de las mujeres cuidadoras con procesos crónicos de salud de familiares dependientes. Aten Primaria. 2016;48(2):77-84. http://dx.doi.org/10.1016/j. aprim.2015.03.006

33. Mosher CE, Adams RN, Helft PR, O’Neil BH, Shahda S, Rattray NA, et al. Positive changes among patients with advanced colorectal cancer and their family caregivers: a qualitative analysis. Psychol Heal. 2017;32(1):94-109. http:// dx.doi.org/10.1080/08870446.2016.1247839 